\title{
Isolation of Uncommon Pasteurella multocida Strains from Cattle in North Central Nigeria
}

\author{
Sugun $\mathrm{MY}^{1^{*}}$, Kwaga $\mathrm{JKP}^{2}$, Kazeem $\mathrm{HM}^{3}$, Ibrahim $\mathrm{NDG}^{3}$ and Turaki AU 4 \\ ${ }^{1}$ Bacteriology Department, National Veterinary Research Institute, Vom, Plateau State, Nigeria \\ ${ }^{2}$ Department of Veterinary Public Health and Preventive Medicine, Ahmadu Bello University, Zaria, Kaduna state, Nigeria \\ ${ }^{3}$ Department of Veterinary Pathology and Microbiology, Ahmadu Bello University, Zaria Kaduna state, Nigeria \\ ${ }^{4}$ Department of Animal Science, Federal University Kashere Gombe. Gombe State, Nigeria
}

*Corresponding author: Sugun MY, Bacteriology Department, National Veterinary Research Institute, Vom, Plateau State, Nigeria, Tel: +2347037712154; E-mail: sugunm@gmail.com

Received date: March 24, 2016; Accepted date: May 09, 2016; Published date: May 20, 2016

Copyright: (C) 2016 Sugun MY, et al. This is an open-access article distributed under the terms of the Creative Commons Attribution License, which permits unrestricted use, distribution, and reproduction in any medium, provided the original author and source are credited.

\begin{abstract}
The prevalence of $P$. multocida, an aetiologic agent of bovine haemorrhagic septicaemia was studied using a purposive sampling technique in north central Nigeria. A total of 18 positive isolates of $P$. multocida were obtained from 175 lungs, liver, and spleen samples examined, giving an isolation rate of $10.3 \%$. Nineteen isolates were confirmed as $P$. multocida by Microbact GNB 24E supplied software version Microbact ${ }^{\mathrm{TM}} 200$ identification package V2.03 (Windows ${ }^{\mathrm{TM}}$ ) and by species specific PCR. By the software interpretations package the percentage probabilities of 12 isolates were above $75 \%$ and 7 others were below $75 \%$. The study confirmed the presence of the African capsular strain E (511 bp) and a unique capsular $F$ type. The $P$. multocida strains were somatically typed as: P. multocida E: 3, 4 and P. multocida E: 2, 5, but most were untypeable. Also of interest is capsular group $\mathrm{F}$ somatically untypeable strain being identified for the first time from calves in Nigeria. These strains have not previously been reported in Nigeria or within the West African sub-region. These could redefine the vaccine strategy as the current vaccine used in Nigeria contain P. multocida B: 3,4 and E: 2 . However more work needs to be carried out in other parts of the country to gather more relevant information with regards to capsular and somatic types.
\end{abstract}

Keywords: Pasteurella multocida; Animals; PCR; Serotypes

\section{Introduction}

The Gram negative bacterium $P$. multocida infects a wide range of animal species, causing diseases such as atrophic rhinitis in pigs [1], fowl cholera in poultry [2] and haemorrhagic septicaemia and shipping fever in cattle $[3,4]$. First reported type-specific capsular antigen in $P$. multocida [5]. Later, there were five distinct capsular serogroups of $P$. multocida (serogroups A, B, D, E and F) indicated [6]. In addition to the capsule serogroups, 16 major somatic serotypes (Identified as 1 through 16) were recognized. Lipopolysaccharides, determined by gel diffusion precipitin test [6] are the antigens that determine somatic type specificity. Various somatic serotypes occur among the different capsule serogroups and current serological nomenclatures designate both of these features.

It is well known that $P$. multocida with certain antigenic compositions can be associated with specific diseases in animals. For example P. multocida B:2 and E2 serotypes cause haemorrhagic septicaemia in cattle. However, a substantial reclassification of bovine relevant Pasteurellaceae has been carried out since 1999, starting with the introduction of the new genus Mannheimia [6]. Consequently, the accuracy of a routine bacterial diagnosis of bovine pasteurellosis, and of the epidemiology and the antimicrobial susceptibilities of Pasteurellaceae based on former investigations is questionable. Thus, there is a need to reinvestigate bovine Pasteurellaceae according to this reclassification assisted by currently indispensable molecular identification tools [7]. Reported a PCR based method for the capsular typing of P. multocida. The capsular PCR is now regarded as the gold standard test [8] and has been used in a number of studies of isolates from a range of animal hosts [9-11]. While the advantage of the PCR based approach has been well recognized, the adoption of PCR-based technologies in Veterinary laboratories in the developing world has been limited in some areas by factors such as cost and technical expertise [12]. Reported that PCR-based typing was more discriminative and could further subtype previously untypeable strains. PCR serotyping is therefore a highly speciesspecific, sensitive and robust method for detection and differentiation of P. multocida serogroups compared to conventional serotyping [13]. Organisms belonging to the bacterial family Pasteurellaceae are ubiquitously present in the respiratory, alimentary and reproductive tracts of different avian, mammalian, reptilian, and likely amphibian hosts [14]. Haemorrhagic septicaemia and mastitis caused by P. multocida is among the important diseases, which in case of mistaken diagnosis can cause a high rate of mortality in cattle [15]. Besides P. multocida types $B: 2$ and $E: 2$ which are the main causes of haemorrhagic septicaemia, many other serotypes viz; B:3, B:4, B:3,4, B:2,5, B:5, E:2,5 have reportedly been encountered in feral and domestic ruminants including cattle, deer, elk and bison [16-18]. The lists of haemorrhagic septicaemia causing strains of P. multocida appear to be widening. There is a dearth of research in this area and this has led to several unresolved questions. For example, besides the so-called African serotype or strain (E: 2), to what extent are new or other serotypes of $P$. multocida involved in haemorrhagic septicaemia outbreaks in Africa. Also to date a meaningful attempt has not been made to relate the field strains and reference vaccine strains, or for effective control of the disease, which strains need to be represented in the vaccine. Both 
documented and anecdotal evidence of outbreaks of haemorrhagic septicaemia in Nigeria and the rest of African continent abound $[3,19]$. Constant surveillance for current serotypes is therefore imperative to contain bovine haemorrhagic septicaemia. The objective of this study was to determine by means of purposive sampling technique the prevalence of $\mathrm{P}$. multocida within visceral organs of cattle from abattoirs located within nine LGAs of Plateau state, Nigeria (Figures 1 and 2).

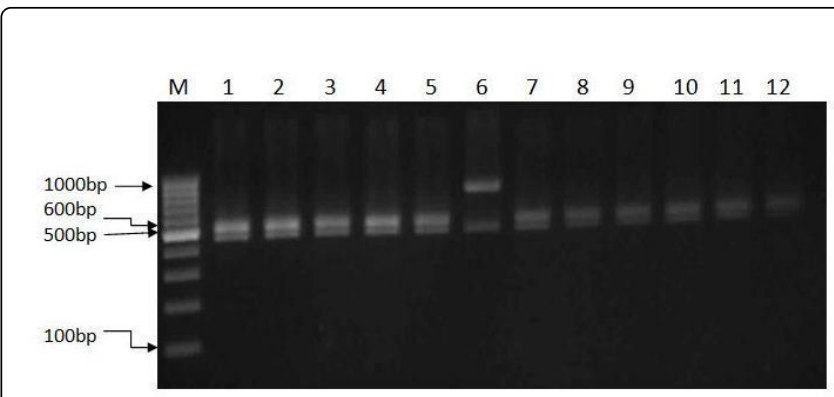

Figure 1: Multiplex PCR profiles with species-specific primers as internal control. Lane 1 (Bld3), Lane 2 (Bld 9) Lane 3 (Bld10), Lane 4 (Jst2), Lane 5 (Js8), lane 6 (Jn3) Lane 7 (Bld3), Lane 8 (Bld3), Lane 9 (Jn6), Lane 10 (Jn12), Lane 11 (Jn14), Lane 12 (Jn18), Lane 12 $(\mathrm{Ka} 2)$ and and lane $\mathrm{M}, 100 \mathrm{bp}$ DNA marker.

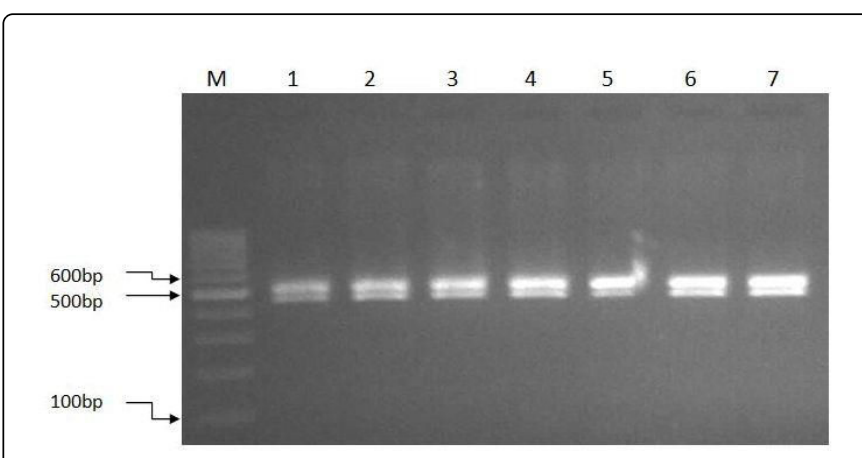

Figure 2: Multiplex PCR profiles with species-specific as internal control Lane 1 (Ka3), Lane 2 (ka4), Lane 3 (ka5), Lane $4(\mathrm{Mg} 4)$, Lane 5 (Mg7), Lane 6 (Ot2), Lane 7 (Jn6) lane M 100 bp DNA molecular size maker.

\section{Materials and Methods}

\section{Sampling}

A total of one hundred and seventy five samples consisting of lungs, liver and spleen were collected from abattoir/slaughter slabs in nine Local Government Area of Plateau state. The samples were placed in sterile bottles, kept in a Coleman box containing ice and transported to the laboratory for examination.

\section{Isolation of Pasteurella multocida}

The tissues were cultured directly on casein sucrose yeast agar (CSY) agar, incubated at $37^{\circ} \mathrm{C}$ for 24 hours [20]. Single non-haemolytic colonies were selected from primary culture and restreaked on fresh Blood agar (BA) plate and incubated at $37^{\circ} \mathrm{C}$ for 24 hours to obtain single colonies of the isolates. The cultures so obtained were subjected to Gram's staining to check for purity of the growth morphology of the organisms and their ability to grow on MacConkey agar (MCA). The isolates which failed to grow on MCA were preliminarily presumed to be $P$. multocida. The cultures were then transferred onto nutrient agar slants for storage, pending further identification by biochemical tests. Further tests included: Oxidase, catalase, indole, citrate utilization, nitrate reduction and fermentation of glucose, mannitol, sucrose, mannose, maltose, arabinose, lactose, dulcitol, salicin, inositol and trehalose using $r$ methods described by Cowan and Steel with modification of using CSY agar [21].

\section{Identification of Pasteurella multocida using Microbact GNB 24E}

Identification of $P$. multocida using Microbact GNB 24E (Oxoid, Basingstoke, UK) was employed as a confirmatory test. Before testing, all isolates were streaked on blood agar containing $5 \%$ bovine blood and incubated at $37^{\circ} \mathrm{C}$ for 24 hours. Sterile normal saline was prepared and $5 \mathrm{ml}$ dispensed into each test tube. Using a sterile loop, 1-3 isolated colonies of the culture were picked and emulsified in the $5 \mathrm{ml}$ sterile saline, which was mixed thoroughly and incubated at $37^{\circ} \mathrm{C}$ for 4 hours. The wells of the individual substrates sets were exposed by cutting the end tag of the sealing strip and slowly peeling backward. The plate was placed in a holding tray and using a sterile Pasteur pipette, four drops of the bacterial suspension were added to each well. Using a sterile pipette, the substrates underlined on the holding tray were overlayed with mineral oil i.e. wells 1, 2, 3, 20 and 24 . The plates were incubated at $37^{\circ} \mathrm{C}$ for 48 hours. The plates were removed from the incubator after 48 hours, the adhesive seal peeled and Nitrate, Kovacs, VP and TDA reagents were added to wells $7,8,10$ and 12 respectively. The results were interpreted as stipulated by the manufacturers and the organisms identified using the software version Microbact ${ }^{\mathrm{TM}} 200$ identification package V2.03 (Windows ${ }^{\mathrm{TM}}$ ).

\section{Somatic serotyping of isolates}

The isolates were shipped to the American National Veterinary Services Laboratories, USDA, AMES, IOWA, USA for somatic typing: The isolates were typed using the procedure of Somatic serotyping [22]. A panel of 16 reference antibodies made against Henddleston reference serotypes 1-16 was used in the typing procedure. Antigen and antisera controls were used in each test. The isolates were further confirmed by PCR using species specific primers: PCR: KMT1T7 5'ATC-CGC-TATTTA-CCC-AGT-GG-3' KMT1SP6 5'-GCT-GTAAAC-GAA-CTC-GCC-AC-3'.

\section{Capsular typing via polymerase chain reaction (PCR)}

The detection of capsular genes by PCR for all serogroups was done according to the method described by [23]. An overnight cell culture grown in Brain Heart Infusion (BHI) broth was harvested by centrifugation at $11,337_{\mathrm{x}} \mathrm{g}$ for $5 \mathrm{~min}$. The cell pellet was resuspended in $100 \mu$ of TE (10 mM Tris; 1 mM EDTA, pH 8.0) buffer and boiled for $10 \mathrm{~min}$, followed by immediate chilling. The cell lysate was centrifuged at $11,337_{\mathrm{xg}}$ for $5 \mathrm{~min}$ and the supernatant was used as the DNA template. The concentration of the DNA template was determined using a spectrophotometer at OD 260/280 nm (Eppendorf, Germany). The oligonucleotide sequences of primers previously published were synthesized by Operon (Germany) (Table 1) [24]. 
Citation: Sugun MY, Kwaga JKP, Kazeem HM, Ibrahim NDG, Turaki AU4 (2016) Isolation of Uncommon Pasteurella multocida Strains from Cattle in North Central Nigeria. J Vaccines Vaccin 7: 320. doi:10.4172/2157-7560.1000320

Page 3 of 5

\begin{tabular}{|c|c|c|c|c|c|}
\hline S.No & Isolatees No & Specimen & OCT INDEX & $\%$ Probability & Somatic serotypes \\
\hline 1 & Bld 3 & Spleen & 515632744 & 96.23 & Untypeable \\
\hline 2 & Jn 12 & Lung & 517632660 & 92.75 & Untypeable \\
\hline 3 & Jn 14 & Lung & 537736600 & 99.19 & Untypeable \\
\hline 4 & Jn 18 & Lung & 526733060 & 97.56 & 3,4 \\
\hline 5 & Ka 2 & Lung & 517622664 & 75.4 & Untypeable \\
\hline 6 & Ka 3 & Lung & 517732560 & 93.56 & Untypeable \\
\hline 7 & Jn 5 & Lung & 517232720 & 85.44 & 2,5 \\
\hline 8 & Jst2 & Liver & 517722700 & 97.14 & Untypeable \\
\hline 9 & Ot2 & Lung & 517720700 & 99.35 & Untypeable \\
\hline 10 & Bld 9 & Liver & 517722760 & 77.46 & Untypeable \\
\hline 11 & $\mathrm{Mg} 7$ & Lung & 577737500 & 91.45 & Untypeable \\
\hline 12 & Bld 10 & gnuL & 517722700 & 97.14 & Untypeable \\
\hline 13 & Jn 6 & Liver & 517632764 & 66.16 & Untypeable \\
\hline 14 & Jn 3 & lung & 517231760 & 69.77 & 3,4 \\
\hline 15 & Ka 4 & Liver & 513622742 & 56.58 & Untypeable \\
\hline 16 & Js 8 & Lung & 517732750 & 48 & Untypeable \\
\hline 17 & Ka 5 & Liver & 517722764 & 42.96 & Untypeable \\
\hline 18 & Mg 14 & Spleen & 517732750 & 48 & Untypeable \\
\hline
\end{tabular}

Table 1: Percentage of P. multocida isolates with Microbact GNB 24E and Somatic.

\section{Results}

\section{Biochemical Characteristics of Pasteurella multocida}

All isolates were gram negative and positive for catalase, indole production, oxidase, citrate utilization and nitrate reduction. All isolates fermented glucose, mannitol, sucrose, mannose and were negative for maltose, arabinose, lactose, inositol and trehalose. No growth was observed on MCA agar. Isolates were confirmed by Microbact 24E, where 12 of the isolates had a probability percent above $75 \%$ and six other isolates had probability percent below $75 \%$ by the software interpretation package. Out of the 19 positive isolates, $61.1 \%$ were from lungs, $27.7 \%$ were from liver and $11.1 \%$ from spleen. Three somatic serotypes $(3,4.3,4.2,5)$ were identified among the somatic antigens. All the remaining 15 isolates were reported as un-typeable Table 1. Jos North had the highest percentage positive samples of 13.5 $\%$, followed by Kanam L.G.A with $9 \%$. Barkin ladi had a percentage positive sample of $6.8 \%$, Jos south $4.5 \%$, Mangu $4.4 \%$ and Quanpaan $2.2 \%$. Langtang North, Ryom and Pankshin did not have any positive samples.

\section{Discussion}

Pasteurella multocida is an important pathogen causing a number of diseases in various domestic and wild animals and avian species. The most important diseases are haemorrhagic septicaemia and septicaemic pasteurellosis in cattle and buffaloes, pneumonia and septicaemic pasteurellosis in sheep and goats, pneumonia, atrophic rhinitis and septicaemia in pigs and fowl cholera or avian cholera in poultry/turkey resulting in heavy economic losses [25]. However, the distribution and prevalence of $P$. multocida serotypes and pathotypes can vary considerably from region to region and over time in a given region. Contemporary studies aimed at unraveling or defining the current spectrum of $P$. multocida serotypes in cattle in Nigeria are lacking. The overall isolation percentage of $P$. multocida from the total number of samples examined was $10.3 \%$ (Table 1). The various characteristics of $P$. multocida isolated during the present investigation are in accordance with the findings of Francis and Carter who reported the isolation of $17.5 \%$ P. multocida from calves in Zambia [26]. Variation in the characteristic of $P$. multocida has been reported by different authors; for example [27] found variability in oxidase test. Researchers who collected samples from cattle with frank clinical signs of haemorrhagic septicaemia or directly from lungs with obvious pathological lesions reported high incidence of $P$. multocida, including Kielstein \& Schimmel, who reported an isolation rate of 50.4\% in 115 calves with pneumonia in ten herds and [27] also reported an incidence of $25.2 \%$ in samples collected from lung lesions. The variability observed in this study might be due to geographical variation in prevalence and use of chemotherapeutic agents, which may influence the occurrence. Distribution of serogroups by multiplex PCR was determined. Serogroup E was found in $4.4 \%$ samples and serogroup $\mathrm{F}$ in $5.6 \%$ samples examined. Isolates were also typed 
somatically. A notable feature of the current study was the preponderence of untypeable strains. It is interesting that although untypeable strains are generally uncommon in cattle, $83.3 \%$ of isolates were somatically untypeable and only $16.7 \%$ were typeable. It is not known whether the presence of untypeable isolates were attributable to new strains that are not included among the 16 Handlestone reference samples or due to loss of somatic antigens by the isolates. During the study period, other bacterial species, including Haemophilus parasuis and Streptococcus suis, were often co-isolated with pathogenic $P$. multocida strains from the same samples. Although it is not straightforward to distinguish whether $P$. multocida is a primary or a secondary pathogen in mixed infections, the fact that various bacterial species may coexist in a given herd should be considered when attempts are made to control disease outbreaks [28]. Pasteurella multocida serotypes B:2 (6:B) and E:2 (6:E) are the principal causes of HS. Although serotype B: has been mainly reported in Asian countries and E:2 in African countries [20-23], both serotypes have been recovered from the disease in some African countries [23]. Beside type $B: 2$, several other $B$ serotypes (B:3, B:4, and $B: 3,4)$ have been incriminated in recent years in sporadic outbreaks of HS in cattle and feral ruminants such as deer, elk and bison [11]. In Africa, serotype E:2 appears to be dominant with cattle rather than water buffaloes being mainly involved. In the present study, the African strain E: 2,5; E:3,4 and a unique strain $\mathrm{F}$ which somatically was untypeable were encountered.

\section{Conclusion}

In conclusion, haemorrhagic septicaemia is a major disease of cattle in Africa caused by P. multocida serotypes. The disease has been a long standing problem with outbreaks of the disease occurring an annual basis. Although vaccination of cattle has long been the hub of control programe, haemorrhagic septicaemia which is an OIE- listed disease and second to contagious bovine pleuropneumonia in its devastation of cattle in sub-saharan Africa has not been adequately controlled partly due to available vaccines which are undefined and of variable efficacy. Over the years, reports of haemorrhagic septicaemia in Nigeria have been inconclusive as information on the specific serotypes of $P$. multocida involved is seldom determined. It is also not known whether the so-called untypeable strains are also involved in haemorrhagic septicaemia outbreaks. The present study has evidently shown the existence in Nigeria of hitherto unencountered serotypes of P. multocida. The mounting evidence of several more serotypes is a challenge as it has limited our ability to control adequately haemorrhagic septicaemia resulting from infection with $P$. multocida. More epidemiologic studies are however advocated and appropriate comparative transmission studies are underway to determine the pathogenicity of these uncommon strains isolated. These results of this study may influence the redefinition of the vaccine strategy as the current vaccine used in Nigeria contains $P$. multocida B:3,4 and E:2. Furthermore more work needs to be carried out in other parts of the country in order to gather more relevant information with regards to capsular and somatic types.

\section{Acknowledgements}

The authors wish to acknowledge National Veterinary Research Institute Vom for support and permission to publish this research work

\section{References}

1. Chanter N, Rutter JM (1989) Pasteurellosis in pigs and the determinants of virulence of toxigenic Pasteurella multocida. In: Pasteurella and Pasteurellosis, Academic Press, London, pp: 161-195.

2. Glisson JR, Hofacre CL, Christensen JP (2003) Fowl cholera. In: Diseases of Poultry, Ames: Iowa State University Press, pp: 658-676.

3. Carter GR, DE Alwis MCL (1989) Haemorrhagic septicaemia. In: Pasteurella and pasteurellosis, Academic Press, London, UK, pp: 131-160.

4. Frank GH (1989) Pasteurellosis of cattle. In: Pasteurella and Pasteurellosis, Academic Press, London, UK, pp: 197-222.

5. CARTER GR (1955) Studies on Pasteurella multocida. I. A hemagglutination test for the identification of serological types. Am J Vet Res 16: 481-484.

6. Rimler RB, Rhoades KR (1989) Pasteurella multocida In: Pasteurella and pasteurellosis, Academic Press, London, pp: 37-74.

7. Angen $\varnothing$, Ahrens P, Bisgaard M (2002) Phenotypic and genotypic characterization of Mannheimia (Pasteurella) haemolytica-like strains isolated from diseased animals in Denmark. Vet Microbiol 84: 103-114.

8. Dziva F, Muhairwa AP, Bisgaard M, Christensen H (2008) Diagnostic and typing options for investigating diseases associated with Pasteurella multocida. Vet Microbiol 128: 1-22.

9. Hossam GZH (1998) Bacteriological studies on P. multocida in domestic animals in Egypt. Food and Agricultural Organization of United Nations, pp: 222.

10. Varga Z1, Sellyei B, Magyar T (2007) Phenotypic and genotypic characterisation of Pasteurella multocida strains isolated from pigs in Hungary. Acta Vet Hung 55: 425-434.

11. Arumugam ND, Ajam N, Blackall PJ, Asiah NM, Ramlan M (2011) Capsular serotyping of Pasteurella multocida from various animal hosts. A comparison of phenotypic and genotypic methods. Trop Biomed 28: 55-63.

12. Christensen H, Bisgaard M, Aalbaek B, Olsen JE (2004) Reclassification of Bisgaard taxon 33, with proposal of Volucribacter psittacicida gen. nov., sp. nov. and Volucribacter amazonae sp. nov. as new members of the Pasteurellaceae. Int J Syst Evol Microbiol 54: 813-818.

13. Araghy SA (2007) Etiological study of calf pneumonia by analysis of bronchoalveolar fluid. University of Tehran, pp: 30-32.

14. Rimler RB (2000) Restriction endonuclease analysis using Hhal and Hpall to discriminate among group B Pasteurella multocida associated with haemorrhagic septicaemia. J Med Microbiol 49: 81-87.

15. Kumar AA, Shivachandra SB, Biswas A, Singh VP, Singh VP, et al. (2004) Prevalent serotypes of Pasteurella multocida isolated from different animal and avian species in India. Vet Res Commun 28: 657-667.

16. Munir R, Farooq U, Badar N, Khanum A, Wadood A (2006) Restriction endonuclease analysis of P. multocida field isolates by Hha-I. Pakistan Veterinary Journal 26: 80-84.

17. Anosa VO, Isoun (1975) An Outbreak of Haemorhagic septicaemia in Holstein cattle in Nigeria. Possible role of associated factors. Bull. Anim. Hlth and Prod Afri 23: 337-340.

18. Lane EP, Kock ND, Hill FW, Mohan K (1992) An outbreak of haemorrhagic septicaemia (septicaemic pasteurellosis) in cattle in Zimbabwe. Trop Anim Health Prod 24: 97-102.

19. Martrenchar A, Njanpop BM (1994) [First case of an outbreak of hemorrhagic septicemia caused by Pasteurella multocida serotype B6 in northern Cameroon]. Rev Elev Med Vet Pays Trop 47: 19-20.

20. Kwaga JKP, Ekundayo SO, Chuku A, Yusuf AF, Mwankon ES, (2013) Characterization of Pasteurella multocida isolated from chickens in Plateau state. Nig Vet J 34: 765-774.

21. Cowan ST, Steel KJ (1985) Manual for the identification of medical bacteria. Cambridge University Press, London pp:127.

22. Heddleston KL, Gallagher JE, Rebers PA (1972) Fowl cholera: gel diffusion precipitin test for serotyping Pasteruella multocida from avian species. Avian Dis 16: 925-936. 
Citation: Sugun MY, Kwaga JKP, Kazeem HM, Ibrahim NDG, Turaki AU4 (2016) Isolation of Uncommon Pasteurella multocida Strains from Cattle in North Central Nigeria. J Vaccines Vaccin 7: 320. doi:10.4172/2157-7560.1000320

Page 5 of 5

23. Townsend KM, Boyce JD, Chung JY, Frost AJ, Adler B (2001) Genetic Geneticorganization of Pasteurella multocida cap Loci and development of a multiplex capsular PCR typing system. J Clin Microbiol 39: 924-929.

24. De Alwis MCL (1996) Haemorrhagic septicaemia: Clinical and epidemiological features of the disease. Int Workshop on diagnosis and control of HS Bali. Indonesia, pp: 28-30.

25. Francis BK, Schels HF, Carter OR (1980) Type E Pasteurella multocida associated with haemorrhagic septicaemia in Zambia. Vet Rec 107: 135.

26. Varga Z, Sellyei B, Magyar T (2007) Phenotypic and genotypic characterisation of Pasteurella multocida strains isolated from pigs in Hungary. Acta Vet Hung 55: 425-434.
27. Hossam GZH (1998) Bacteriological studies on P. multocida in domestic animals in Egypt. P. H.D. Thesis, Microbiology Faculty of Veterinary Medicine Cairo University, Egypt pp: 222.

28. Cai X, Chen H, Blackall PJ, Yin Z, Wang L, et al. (2005) Serological characterization of Haemophilus parasuis isolates from China. Vet Microbiol 111: 231-236. 